\title{
Vertical Discrepancy in Height of Morse Cone Abutments Submitted to Different Torque Forces
}

\author{
Bruno Q. S. Cordeiro ${ }^{1}$, Carlos Fernando de Almeida Barros Mourão $2,3, * \mathbb{C}$, Waldimir R. Carvalho ${ }^{4}$, \\ Edgard M. Fonseca ${ }^{4}$, Pietro Montemezzi ${ }^{5}$, Kayvon Javid ${ }^{1}{ }^{(}$, , Cintia C.P. Martins ${ }^{4}$, Valquiria Quinelato ${ }^{4} \mathbb{D}^{\circ}$, \\ Mylena D. Moreno ${ }^{4}$ and Priscila L. Casado ${ }^{4, *}$
}

1 Graduate Program in Dentistry, Universidade Federal Fluminense, Niteroi 24020-140, RJ, Brazil; brunoqsc@gmail.com (B.Q.S.C.); onecure@aol.com (K.J.)

2 Clinical Research Unit of the Antonio Pedro Hospital, Universidade Federal Fluminense, Niterói 24033-900, RJ, Brazil

3 Clinical Research Laboratory in Dentistry, Universidade Federal Fluminense, Niteroi 24020-140, RJ, Brazil

4 Implant Dentistry Department, Dentistry School, Universidade Federal Fluminense, Niteroi 24020-140, RJ, Brazil; wcarvalho@id.uff.br (W.R.C.); edgardfonseca@vm.uff.br (E.M.F.); ccpm.odonto@gmail.com (C.C.P.M.); valquiriaquinelato@yahoo.com.br (V.Q.); mylenamoreno@id.uff.br (M.D.M.)

5 Independent Researcher, 24128 Bergamo, Italy; m.montemezzi@libero.it

* Correspondence: mouraocf@gmail.com (C.F.d.A.B.M.); priscilacasado@id.uff.br (P.L.C.); Tel.: +1-941-(830)-1302 (C.F.d.A.B.M.); +55-21-2629-9920 (P.L.C.)

\section{check for} updates

Citation: Cordeiro, B.Q.S.; Mourão, C.F.d.A.B.; Carvalho, W.R.; Fonseca, E.M.; Montemezzi, P.; Javid, K.; Martins, C.C.; Quinelato, V.; Moreno, M.D.; Casado, P.L. Vertical Discrepancy in Height of Morse Cone Abutments Submitted to Different Torque Forces. Materials 2021, 14, 4950. https://doi.org/10.3390/ ma14174950

Academic Editor: Bruno Chrcanovic

Received: 1 July 2021

Accepted: 23 August 2021

Published: 30 August 2021

Publisher's Note: MDPI stays neutral with regard to jurisdictional claims in published maps and institutional affiliations.

Copyright: (c) 2021 by the authors. Licensee MDPI, Basel, Switzerland. This article is an open access article distributed under the terms and conditions of the Creative Commons Attribution (CC BY) license (https:// creativecommons.org/licenses/by/ $4.0 /)$.
Abstract: The present study aimed to evaluate the influence of manual torque $(10 \mathrm{Ncm})$ versus clinical torque $(30 \mathrm{Ncm})$, which is recommended by the manufacturer, on the total length of morse cone implant abutments. Twenty specimens were prepared and distributed into two groups: group 1 with ten analogs for morse cone type implant, and group 2 with ten morse type implants, size $4.3 \times 15 \mathrm{~cm}$. In each group, the distance between the implant platform to the top of the prosthetic abutment (abutment height) was measured and subjected to a torque of $10 \mathrm{Ncm}$. Then, the $30 \mathrm{Ncm}$ torque was applied to the same abutment, and abutment height was measured. The distance between the top of the abutment and the implant/analog base was measured. In order to verify the clinical reproducibility of the experiment, comparisons between the abutment height of the analog at $10 \mathrm{Ncm}$ and the implant at $30 \mathrm{Ncm}$ were performed, showing a greater discrepancy in torque for the $10 \mathrm{Ncm}$ analog $(p<0.05)$. In order to verify if the change in the laboratory protocol from 10 to $30 \mathrm{Ncm}$ could minimize the differences in the height of the prosthetic abutments, the abutment height in groups 1 and 2 was compared with $30 \mathrm{Ncm}$, and no significant difference was observed $(p>0.05)$. The data indicated that the manual torque and the torque recommended by the manufacturer influence the total length of the prosthetic abutments of morse cone implants.

Keywords: morse cone; torque; abutment; implant-supported prosthesis

\section{Introduction}

Among many factors, the success of implant-supported rehabilitations depends on the configuration of the connection between the abutment and the implant platform, as it influences the stability of the prosthesis, the mechanical strength of the implant, and the prosthetic components [1]. For this, the professional should be aware that clinical procedures such as molding, transfer seating, and overload applied to the connection can lead to changes in the implant components which, as consequence, trigger modification in peri-implant tissues, decreasing implant success in the long term [2].

In the lab and oral cavity, the abutment is connected to the implant by a manual torque, while at the final prosthesis connection, the abutment will be connected to the implant to the proper torque value, which is the value of torque recommended by the manufacture [3]. Improper connection may cause interference that results in a lack of stability and prosthetic 
functionality. Therefore, the fit of an implant abutment superstructure, without any interference from manual torque during prosthesis confection to final torque, is essential to the success of the prosthesis [3].

In this context, a source of error when using an internal implant connection could arise from the level of the tightening torque applied to the prosthesis parts by the laboratory technician compared to that applied by the clinician in oral cavity [3]. However, this topic is not clearly addressed in the current literature, and information on the laboratory and clinical differences of prosthetic components of implants is still limited, requiring further studies in order to clarify and minimize prosthesis misfit.

All connections are known to have an amount of maladaptation and bacterial infiltrate, varying according to the type of implant platform [4]. However, the morse cone system seems to behave better, presenting excellent adaptation and less bacterial invasion when compared to other implant systems [4]. This is because the morse cone system manufacturers recommend the installation of the underlying implant at the level of the bone crest, which, usually, allows the maintenance of the peri-implant bone above the implant-abutment junction, even after loading [5]. The implant-abutment interface for morse cone connection could have a minimal or an absence of gap. This allows for bone growth in this space in the implant-abutment interface, establishing the contact between bone and implant during the stabilization of the implant pillar [6], which can reduce future damage to peri-implant tissue.

Since crown misalignment and adaptation between implant and abutment could impact the clinical performance of implant-supported prosthesis, it is up to the professional to understand the stages of the screw-implant prosthesis connection [7]. To minimize the screw loosening, it is documented in the literature that the screw should be retightened at least twice at 10-min intervals in all laboratory and clinical procedures [8]. For this, the use of specific wrenches is indicated along with the specific mechanical torque, following the manufacturer's indications for the type of prosthetic abutment [9]. However, this procedure in the laboratory phase is usually not feasible. The need to remove the prosthetic component for clinical installation may cause physical changes and discrepancy of forces applied during implant torque and the tightening process [10].

Adaptation of the implant-supported prosthesis is crucial for the longevity of endosseous implant rehabilitation treatment. There are many factors that may interfere with prosthetic stability that can directly influence the development of future clinical protocols. Therefore, it is important to determine the vertical discrepancies between non-torqued and torqued abutments, since it is directly associated with occlusal contact adjustments in implant crowns and implant success in the long term. However, the scarcity of studies regarding clinical and laboratory aspects focusing on forces applied to prosthetic connection can lead to a gap in the basic knowledge related to making and adaptation of the prosthesis. Therefore, the objective of this study is to evaluate the discrepancy in axial displacement of morse cone implant abutments when compared to manual torque in the laboratory with that recommended by the manufacturer in oral cavity. Our hypothesis is that after torque application during prosthesis confection, there are discrepancies in the abutment implant complex.

\section{Materials and Methods}

\subsection{Research Design}

This research is of a technical laboratory nature, not involving the participation of humans or animals, or any procedure that includes biological material or personal data; therefore, it is not necessary to submit this research for evaluation by the Research Ethics Committee.

Research Instrument: In this study, 20 specimens were made for further division into 2 groups: group 1 with 10 analogs for morse cone implant type (Conexão, Implant Systems, São Paulo, Brazil), numbered 1 to 10; and group 2 with 10 implants, morse cone type, with size $4.3 \times 15$ cm (Conexão, Implant Systems, São Paulo, Brazil), numbered from 11 to 20 . 
All implants or analogs were installed using the same methodology: inside resin cylinders, density $1.42 \mathrm{~g} / \mathrm{cm}^{3}$, with elastic modulus greater than $3 \mathrm{GPa}$ (Polyacetal, Caterplast, São Paulo, Brazil) in type IV durone stone plaster (Dentsply, Dentsply Ind. Com., Rio de Janeiro, Brazil).

A $7 \mathrm{~mm}$ diameter and $17 \mathrm{~mm}$ deep guide hole was drilled into the resin cylinder to standardize the fixation of implants/analogs and the amount of plaster used so that implants and analogs would not move when the abutment attached to them was torqued (Figure 1). Twenty $9.0 \mathrm{~mm}$ preparation abutments (Conexão, Implant Systems, São Paulo, Brazil) screwed with the Torq Control ${ }^{\circledR}$ universal torque wrench (Anthogyr PT, Sallanches, France) were used on each implant and analog of both groups.

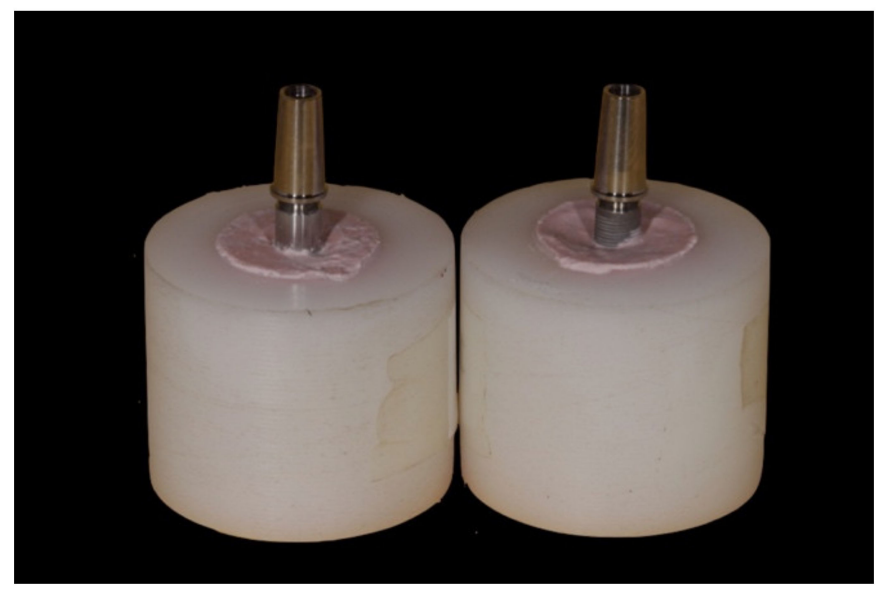

Figure 1. Resin cylinder to standardize the fixation of analogs (left)/implants (right).

Each pillar was subjected to the same torque force between the groups: $10 \mathrm{Ncm}$ and $30 \mathrm{Ncm}$, presenting at the end the following division of groups: group 1 (analog; $\mathrm{n}=10$ ): $10 \mathrm{~N}$ and $30 \mathrm{~N}$; group 2 (implant; $\mathrm{n}=10$ ): $10 \mathrm{Ncm}$ and $30 \mathrm{Ncm}$. In each group, the abutment was subjected to the torque of $10 \mathrm{Ncm}$, and the first distance A-B was measured. Then, the $30 \mathrm{~N}$ torque was applied to the same abutment, and the second measurement $\mathrm{A}-\mathrm{B}$ was made (Figure 2).

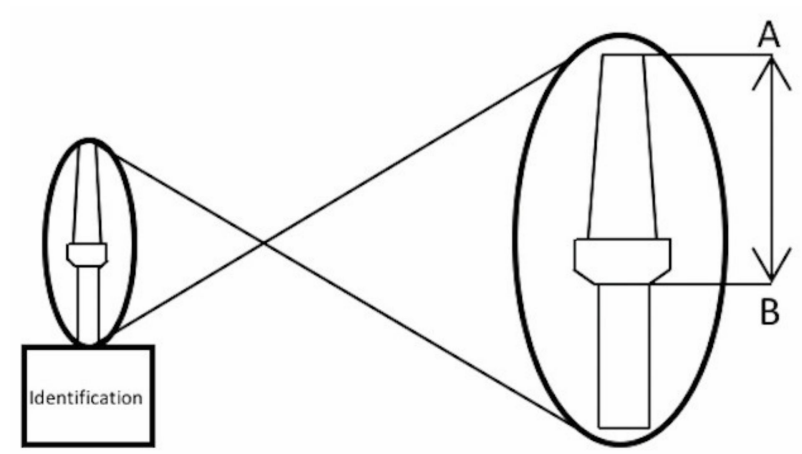

Figure 2. A-B distance ( $\mathrm{mm})$. A: A-B distance (distance between the implant platform to the top of the abutment). The letter A represents the maximum height of the abutment and the letter $B$ represents the base of the implant.

The distance between the top of the abutment and the implant/analog base (A-B) was measured at the Brazil Mitutoyo Calibration Laboratory, accredited by CGCRE according to ABNT NBRI ISO/IEC 17025, under number CAL0031, with a measurement uncertainty of $0.003 \mathrm{~mm}$, a coverage factor $(\mathrm{k})$ of 2.00 , and infinite degrees of freedom. 


\subsection{Statistical Analysis}

The measurements obtained in groups 1 and 2, with different torques of $10 \mathrm{~N}$ and $30 \mathrm{~N}$, were tabulated and subjected to qualitative statistics, considering the median and standard deviation of the numerical variables evaluated. Microsoft Office 2013 Excel was used for data tabulation and Prisma GraphPad 6.0 software was used for statistical calculations and graphic production. The sample size was based on previous studies $[7,8]$, considering the power of $80 \%$.

The paired $t$-test correlated the measurements of the variables studied within each group, after verifying the distribution normality, with a significance level of $0.05 \%$. Values of $p<0.05$ were considered significant.

\section{Results}

The results considered the statistical evaluation between the $10 \mathrm{~N}$ and $30 \mathrm{~N}$ torques, within each group and between groups.

Result of the general analysis of groups 1 and 2: The results, in mm (distance A-B), are listed below (Table 1) for the $10 \mathrm{Ncm}$ and $30 \mathrm{Ncm}$ values.

Table 1. Individual values of distances between points A and B ( $\mathrm{mm})$.

\begin{tabular}{|c|c|c|}
\hline & $10 \mathrm{Ncm}$ & $30 \mathrm{Ncm}$ \\
\hline \multirow{10}{*}{ Analogs (Group 1) } & 9.507 & 9.497 \\
\hline & 9.497 & 9.479 \\
\hline & 9.485 & 9.470 \\
\hline & 9.515 & 9.498 \\
\hline & 9.491 & 9.478 \\
\hline & 9.511 & 9.494 \\
\hline & 9.487 & 9.470 \\
\hline & 9.503 & 9.505 \\
\hline & 9.485 & 9.470 \\
\hline & 9.536 & 9.514 \\
\hline Minimum value & 9.485 & 9.470 \\
\hline Maximum value & 9.536 & 9.514 \\
\hline Mean \pm SD & $9.5017 \pm 0.016$ & $9.487 \pm 0.016$ \\
\hline \multirow{10}{*}{ Implants (Group 2) } & 9.503 & 9.480 \\
\hline & 9.487 & 9.468 \\
\hline & 9.493 & 9.474 \\
\hline & 9.463 & 9.437 \\
\hline & 9.485 & 9.47 \\
\hline & 9.466 & 9.441 \\
\hline & 9.527 & 9.504 \\
\hline & 9.488 & 9.462 \\
\hline & 9.555 & 9.538 \\
\hline & 9.503 & 9.488 \\
\hline Minimum value & 9.463 & 9.437 \\
\hline Maximum value & 9.555 & 9.538 \\
\hline Mean \pm SD & $9.497 \pm 0.027$ & $9.476 \pm 0.029$ \\
\hline
\end{tabular}

Groups 1 and 2 presented normal distribution of data after the Shapiro-Wilk test $(p>0.05)$, so the paired $t$-test was used.

\subsection{Analog Results}

Considering the comparative analysis between the $10 \mathrm{Ncm}$ and $30 \mathrm{Ncm}$ torques in group 1 (analogs), it can be observed that there is a statistically significant difference between the distances $\mathrm{A}-\mathrm{B}$, with a greater distance in the $10 \mathrm{Ncm}$ torque (Figure 3). 


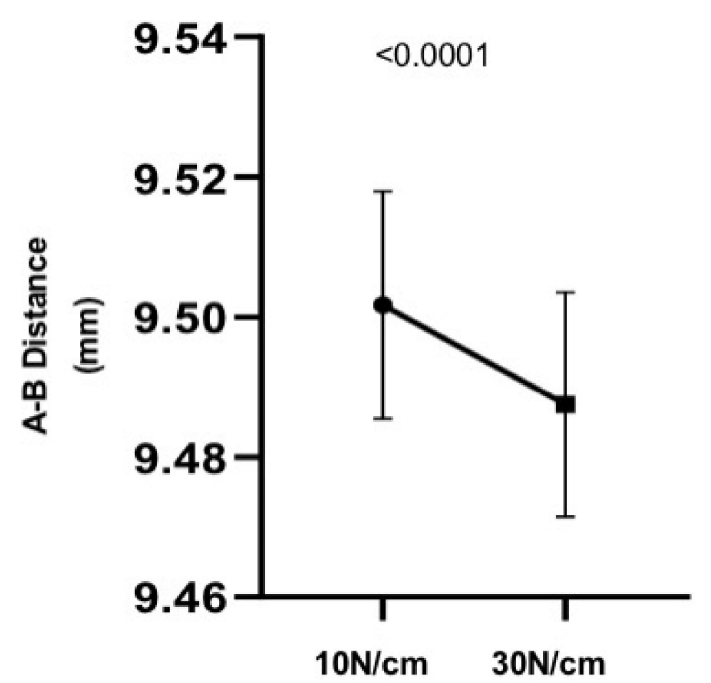

Figure 3. Chart showing a significant decrease in A-B distance between $10 \mathrm{~N}$ and $30 \mathrm{~N}$ in the pillars on the analogs (Group 1).

\subsection{Implants Results}

Considering the comparative analysis between the $10 \mathrm{~N}$ and $30 \mathrm{~N}$ torques in group 2, it can be observed that there is a statistically significant difference between the distances $\mathrm{A}-\mathrm{B}$, with a greater distance in the $10 \mathrm{~N}$ torque (Figure 4 ).

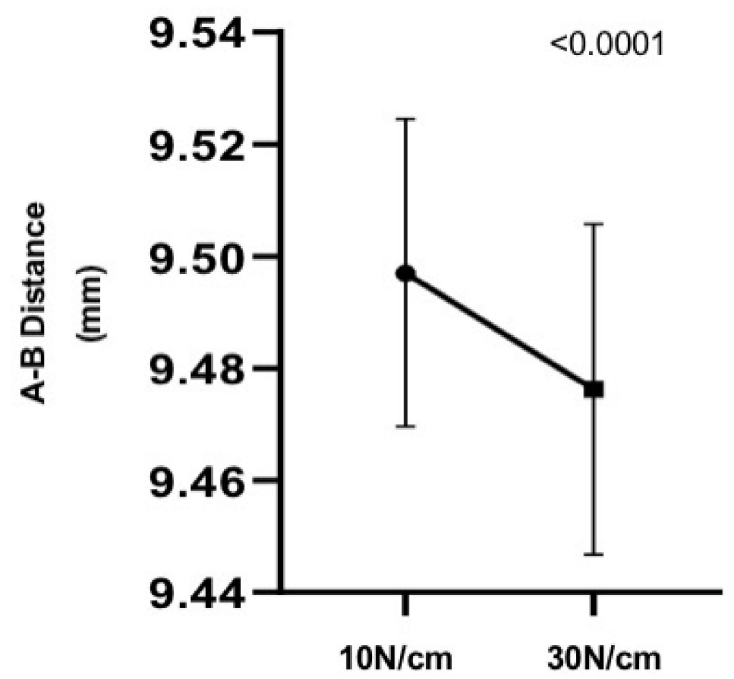

Figure 4. Chart showing a significant decrease in A-B distance between $10 \mathrm{~N}$ and $30 \mathrm{~N}$ in the pillars on the implants (Group 2).

\section{3. $10 \mathrm{Ncm}$ Analog and $30 \mathrm{Ncm}$ Implant Comparison}

In order to verify the clinical reproducibility of the experiment, comparisons between A-B measurements on the analog at $10 \mathrm{Ncm}$ and implant at $30 \mathrm{Ncm}$ were performed. The results showed a statistically significant difference, with greater A-B distance in the $10 \mathrm{Ncm}$ torque in the analog $(p<0.05)$ (Figure 5).

\section{4. $30 \mathrm{~N}$ Analog and $30 \mathrm{~N}$ Implant Comparison}

In order to verify if the change in the laboratory protocol from 10 to $30 \mathrm{~N}$ could minimize the differences in the heights of the prosthetic abutments, the A-B distance between the analogs $(30 \mathrm{~N})$ and implants $(30 \mathrm{~N})$ was compared. There was no statistically significant difference between the groups for this torque $(p>0.05)$ (Figure 6). 


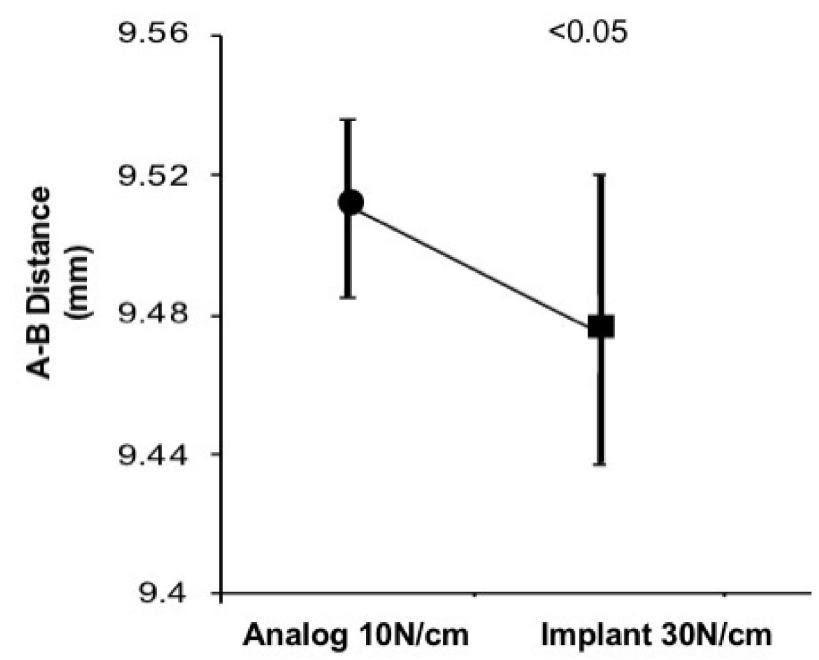

Figure 5. Chart showing significant decrease in A-B distance between $10 \mathrm{Ncm}$ (analog) and $30 \mathrm{Ncm}$ (implant).

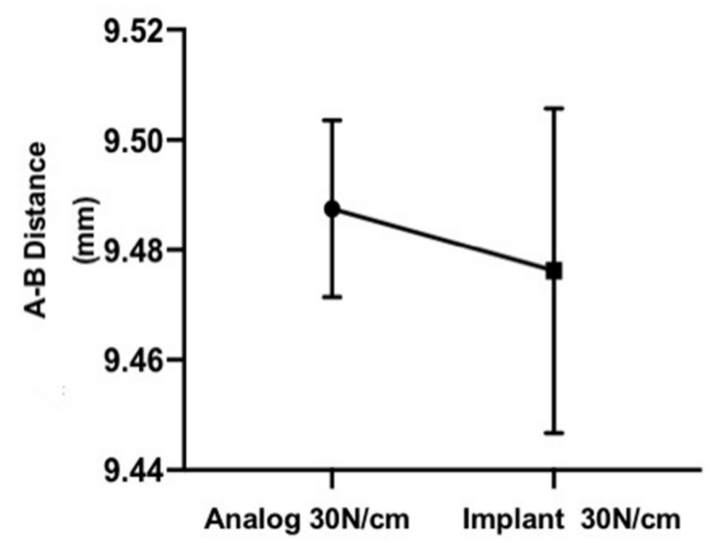

Figure 6. Chart showing the similarity between the distance A-B when the $30 \mathrm{~N}$ torque is applied to the analog and the implant.

\section{Discussion}

Despite the long-term success associated with endosseous implant rehabilitation, complications related to mechanical and biological aspects still have high rates [9]. Mechanical failures represent about $60-80 \%$ of complications in implant dentistry [11], being one of the main problems related to fixed prosthesis failures. It is noteworthy that the discrepancy in the adaptation of prosthesis from the prosthetic laboratory to the adaptation in the patient's oral cavity may be a decisive factor for the longevity of treatment. Therefore, this work aimed to evaluate the influence of manual torque force and the force recommended by the manufacturer on the total lengths of morse cone implant abutments. Our results showed that there is a difference in the heights of the prosthetic abutments when using the manual torque of $10 \mathrm{Ncm}$ and the clinical torque of $30 \mathrm{~N}$. The discrepant decrease in the distance between the implant platform to the top of the abutment was observed in both groups. However, when comparing prosthetic abutments supported on both analogs and implants with the same torque $(30 \mathrm{Ncm})$, there was no difference in the height of the prosthetic abutment, which demonstrates that the difference in prosthesis height is related to the torque force and not to the torque component connected to the abutment.

The morse cone implants have a strong imbrication between the internal surface of the implant and the prosthetic component, leading to less movement between these structures and helping to prevent the passage of microorganisms from within the implant $[12,13]$. Therefore, in this research, the morse cone implant was selected to allow 
these results to be extrapolated to other types of connections that demonstrate greater prosthetic instability. However, our study showed that even in the presence of the characteristic clamping of this connection, when different torque forces are applied, a vertical displacement of the abutment can be promoted, and there can be possible space creation in the prosthesis components.

The presence of spaces between implant components is at great risk for the spread of a bacterial reservoir, accentuating possible peri-implant soft tissue inflammation and culminating in the development of mucositis [14,15], which is today characterized as a highly incident disease in the population rehabilitated with endosseous implants [16]. This disease is closely related to long-term success in implantology, as mucositis is the obvious precursor of peri-implantitis, which is a major cause of implant loss [17]. Thus, preventing the creation of prosthetic mismatches may be of great relevance to implant survival.

Another important aspect to consider is the torque applied to the prosthetic component of the implant. In theory, the torque should follow the manufacturer's instructions. However, during fabrication of the prosthesis in the laboratory, the component is usually fitted over the analog, which simulates the implant by manual torque. Thus, two main problems can be generated: a lower torque during prosthesis making and prosthesis adaptation in an analog, not in an endosseous implant.

Al-Otaibi et al. [8] conducted a study comparing the effect of different applications of torque on implant-supported fixed unit prosthesis, showing that torsion may influence prosthesis adjustment and implant survival. Studies show that manual torque, often applied during prosthesis fabrication, reaches a maximum of $20 \mathrm{Ncm}$ and has torque differences in about $48 \%$ of the prosthesis fabricated $[18,19]$.

According to the study by Dellinges and Tebrock, in 1993 [2], screws that require more than $10 \mathrm{Ncm}$ of torque cannot be tightened manually using commercially available hand wrenches. Therefore, mechanical or electrical torque control is required. In our study, all specimens were subjected to controlled torque forces in order to make the results replicable and standardized. We observed that the differences between the devices used in the laboratory during the making of the prosthesis, $10 \mathrm{Ncm}$, influence the difference in the height of the prosthetic abutment, which is the distance between the implant platform to the top of the abutment when it is subsequently submitted to the application of $30 \mathrm{Ncm}$ torque.

On the other hand, the use of different torques on analogs and implants did not influence the height of the prosthetic abutment, showing that the use of the analog during the prosthesis fabrication is not directly related to future mismatches associated with the crown height but rather the applied torque. A study by Saber et al. [7] showed the discrepancy of the abutment height between the different types of implant platforms, showing greater discrepancy in the external hexagon system. However, in the present study, we did not consider the simulation of the laboratory phase of prosthesis confection, using analog, as done in our study.

The pattern of discrepancies observed in our study evidenced the decrease in the distance between the implant platform to the top of the abutment, similarly in all analoganalog, analog-implant, and implant-implant comparisons, when subjected to $10 \mathrm{Ncm}$ and $30 \mathrm{Ncm}$ torque. Comparing an "ex vivo" study to the patient's mouth, the abutment in the mouth could generate significant changes in the height of the prosthesis, triggering infraocclusion and related pathologies. For this, future laboratory procedures may be developed, considering these discrepancies and their possible clinical consequences, aiming to minimize the damage to the peri-implant tissues. The presence of discrepancies in laboratory and clinical procedures shows that a correct torque is needed in the laboratorial procedures and then, another new screw is necessary to retain the crown in mouth in future protocols. In addition, future studies are needed, using different types of abutments and implant systems, in an attempt to ratify the applicability of the results found. In addition, the authors suggest exploring ways to minimize this discrepancy regarding its possible clinical issues. 


\title{
5. Conclusions
}

This laboratory study concluded that there is a change in the distance between the implant platform to the top of the prosthetic abutment with different torques $(p<0.001)$, which is representative of the laboratory confection and clinical application of the unit prosthesis on morse cone implants.

\begin{abstract}
Author Contributions: B.Q.S.C.: conceptualization, investigation, methodology, software, writingoriginal draft preparation; C.F.d.A.B.M.: investigation, methodology, validation, supervision, writingreview and editing; funding acquisition; W.R.C.: investigation, methodology; data curation; validation, supervision; writing — original draft preparation; E.M.F.: investigation, methodology; data curation; validation, supervision; writing — original draft preparation; P.M.: investigation, validation, funding acquisition; K.J.: investigation, validation; writing — review and editing; C.C.P.M.: investigation, validation; V.Q.: investigation, validation; M.D.M.: investigation, validation; P.L.C.: conceptualization, investigation, methodology, supervision, writing-review and editing. All authors have read and agreed to the published version of the manuscript.
\end{abstract}

Funding: All funding was provided by the Implant Dentistry Post-graduation of Universidade Federal Fluminense.

Institutional Review Board Statement: Not Applicable.

Informed Consent Statement: Not Applicable.

Data Availability Statement: Data sharing is not applicable to this article.

Conflicts of Interest: The authors declare no conflict of interest.

\section{References}

1. Rezende, C.E.E.; Brisola, A.O.P. Conexões implante/pilar em implantodontia. Innov. Implant. J. Biomater. Esthet. 2014, 9, 58-64.

2. Dellinges, M.A.; Tebrock, O.C. A measurement of torque values obtained with hand-held drivers in a simulated clincal setting. J. Prosthodont. 1993, 2, 212-214. [CrossRef] [PubMed]

3. Dailey, B.; Jordan, L.; Blind, O.; Tavernier, B. Axial displacement of abutments into implants and replicas, with the tapered cone-screw internal connection, as a function of tightening torque. Int. J. Oral Maxillofac. Implant. 2009, 24, 251-256.

4. Ceruso, F.M.; Barnaba, O.; Mazzoleni, S.; Ottria, L.; Gargari, M.; Zuccon, A.; Bruno, G.; Di Fiore, A. Implant-Abutment connection on single crowns: A systematic review. Oral Implantol. 2017, 10, 349-353. [CrossRef] [PubMed]

5. Degidi, M.; Daprile, G.; Piattelli, A. Marginal bone loss around implants with platform-switched Morse-cone connection: A radiographic cross-sectional study. Clin. Oral Implant. Res. 2017, 28, 1108-1112. [CrossRef] [PubMed]

6. Weng, D.; Nagata, M.J.; Bosco, A.F.; de Melo, L.G. Influence of microgap location and configuration on radiographic bone loss around submerged implants: An experimental study in dogs. Int. J. Oral Maxillofac. Implant. 2011, 26, 941-946.

7. Saber, F.S.; Abolfazli, N.; Ataei, S.J.; Motlagh, M.T.; Gharekhani, V. The effect of repeated torque tightening on total lengths of implant abutments in different internal implant-abutment connections. J. Dent. Res. Dent. Clin. Dent. Prospect. 2017, 11, 110-116. [CrossRef] [PubMed]

8. Al-Otaibi, H.N.; Almutairi, A.; Alfarraj, J.; Algesadi, W. The effect of torque application technique on screw preload of implantsupported prostheses. Int. J. Oral Maxillofac. Implant. 2017, 32, 259-263. [CrossRef] [PubMed]

9. Kim, K.S.; Lim, Y.J.; Kim, M.J.; Kwon, H.B.; Yang, J.H.; Lee, J.B.; Yim, S.H. Variation in the total lengths of abutment/implant assemblies generated with a function of applied tightening torque in external and internal implant-abutment connection. Clin. Oral Implant. Res. 2011, 22, 834-839. [CrossRef] [PubMed]

10. Alikhasi, M.; Kazemi, M.; Jalali, H.; Hashemzadeh, S.; Dodangeh, H.; Yilmaz, B. Clinician-generated torque on abutment screws using different hand screwdrivers. J. Prosthet. Dent. 2017, 118, 488-492. [CrossRef]

11. Papaspyridakos, P.; Chen, C.J.; Singh, M.; Weber, H.P.; Gallucci, G.O. Success criteria in implant dentistry: A systematic review. J. Dent. Res. 2012, 91, 242-248. [CrossRef]

12. Norton, M.R. Assessment of cold welding properties of the internal conical interface of two commercially available implant systems. J. Prosthet. Dent. 1999, 81, 159-166. [CrossRef]

13. Assenza, B.; Tripodi, D.; Scarano, A.; Perrotti, V.; Piattelli, A.; Iezzi, G.; D’Ercole, S. Bacterial leakage in implants with different implant-abutment connections: An in vitro study. J. Periodontol. 2012, 83, 491-497. [CrossRef] [PubMed]

14. Orsini, G.; Fanali, S.; Scarano, A.; Petrone, G.; di Silvestro, S.; Piattelli, A. Tissue reactions, fluids, and bacterial infiltration in implants retrieved at autopsy: A case report. Int. J. Oral Maxillofac. Implant. 2000, 15, 283-286.

15. Tsuruta, K.; Ayukawa, Y.; Matsuzaki, T.; Kihara, M.; Koyano, K. The influence of implant-abutment connection on the screw loosening and microleakage. Int. J. Implant. Dent. 2018, 4, 11. [CrossRef] [PubMed]

16. Meyle, J.; Casado, P.; Fourmousis, I.; Kumar, P.; Quirynen, M.; Salvi, G.E. General genetic and acquired risk factors, and prevalence of peri-implant diseases-Consensusreport of working group 1. Int. Dent. J. 2019, 69, 3-6. [CrossRef] [PubMed] 
17. Lang, N.P.; Berglundh, T. Periimplant diseases: Where are we now? Consensus of the Seventh European Workshop on periodontology. J. Clin. Periodontol. 2011, 38, 178-181. [CrossRef]

18. Vallee, M.C.; Conrad, H.J.; Basu, S.; Seong, W.J. Accuracy of friction-style and spring-style mechanical torque limiting devices for dental implants. J. Prosthet. Dent. 2008, 100, 86-92. [CrossRef]

19. McCracken, M.S.; Mitchell, L.; Hegde, R.; Mavalli, M.D. Variability of mechanical torque-limiting devices in clinical service at a US dental school. J. Prosthodont. 2010, 19, 20-24. [CrossRef] [PubMed] 